\title{
]jfis
}

\section{Wearable Walking Care Checking Gait Device Using the AHRS Sensor}

\author{
Nan-Hee Kim ${ }^{1}$, Ju-Hyung Lee ${ }^{1}$, Seo-Jin Woo ${ }^{1}$, Dong-Lin Kwon ${ }^{2}$, and \\ Suk-Gyu Lee ${ }^{3}$ \\ ${ }^{1}$ Department of Electronic Engineering, Yeungnam University, Gyeongsan, Korea \\ ${ }^{2}$ The Kiturami Boiler Company, Cheongdo, Korea \\ ${ }^{3}$ Department of Electrical Engineering, Yeungnam University, Gyeongsan, Korea
}

\begin{abstract}
This paper presents implementation of an attitude and heading reference system (AHRS) based on detachable gait detection device. Recent research has focused on measuring and taking feedback from the gait. However, there is a lack of research on the convenience of the user. So, a detachable gait detection device considering the user's view is proposed. Unlike, traditional insole type sensors, which are applied only to the running shoes. This study makes it possible to apply it, to various shoes other than specific purpose shoes. In particular, we have studied algorithms that can measure various values like gait type, number of steps. That values measured only by the sensors, built in insole, but now we can use detachable type outside the shoe. This algorithm can be used in a straight line as well as any type of pattern you encounter in everyday life. Experiments to prove the performance and accuracy of this algorithm were carried out like, for classifying different walking styles and measuring steps. It is possible to recognize unusual behavior of tread without going to the hospital through this device, and it will reduce the cost and psychological burden of consumers.
\end{abstract}

Keywords: Detachable type, Gait analysis, Toe-in walking, Toe-out walking

Received: Mar. 1, 2019

Revised : Jun. 14, 2019

Accepted: Jun. 21, 2019

Correspondence to: Suk-Gye Lee (sglee@ynu.ac.kr)

(CThe Korean Institute of Intelligent Systems

(c) This is an Open Access article distributed under the terms of the Creative Commons Attribution Non-Commercial License (http://creativecommons.org/licenses/ by-nc/3.0// which permits unrestricted noncommercial use, distribution, and reproduction in any medium, provided the original work is properly cited.

\section{Introduction}

Today, as the quality of life is increasing, the health consciousness is also increasing. In recent years, the number of patients suffering from diseases of the spine, neck, and legs has increased. They become subject of the diseases such as scoliosis, which is a big problem in modern society. These health problems are related to gait. The wrong gait leads to knee and pelvic swaying, deforming the shape of the feet and legs. Not only imbalance of body shape which don't look good but also affect from the pelvis to the neck, spine and joint health. Therefore, it is important to remedy the wrong gait.

The purpose of creating a gait therapy system is important for people's health and medical care. However, this can be financially and psychologically burden for a person to visit the hospital to fix their gait. As a result, recent research has developed a system that provides feedback to correct the gait in real life [1].2]. This feedback system was tested on, inside the insole of the shoes [3-6]. Shoes and watches for gait correction are already in the market. In the case of a watch, it is impossible to distinguish whether the user's walking pattern is toe-in or toe-out. These gait analysis systems are only applicable towards the running shoes. People 
wear shoes with different sizes and shapes, such as dress shoes, sandals, and slippers. The purpose of this study is to develop a system that gives feedback to the user for different footwear. This paper introduces a portable device that can be wear on the outside of the shoe. Proposed algorithm for this wearable device is able to distinguish typical abnormal walking patterns through the use of LED display.

\section{Gait Classification}

Typical abnormal walking patterns contain steady-state walking, toe-in and toe-out walking. Additionally, these gaits are classified by angle. The abnormal gait, toe-out walking is a gait pattern in which the feet spread outward more than 15.0 degrees from the direction towards which the body moves. Furthermore, toe-in walking is determined feet spread more than -13.7 degrees from the direction toward which the body moves. Moreover, this sensor can also measure the gait that appears in both stance and swing phase. Assume that, the sensor is attached on the top side of the right shoe, the angle of walking can be classified. It is also assumed that, the movement of the user's right foot is symmetrical to the left one. Toe-in walking was based on standard deviation from the study of Rosenbaum [7]. Since, toe-out walking was an impossible walking posture when the study by Rosenbaum [7] was conducted, another study of toe-out walking was referenced [8].

Table 1. Gait classification according to angle

\begin{tabular}{cccc}
\hline Gait & $\begin{array}{c}\text { Toe-in } \\
\text { walking }\end{array}$ & $\begin{array}{c}\text { Stable } \\
\text { walking }\end{array}$ & $\begin{array}{c}\text { Toe-out } \\
\text { walking }\end{array}$ \\
\hline Angle (deg) & $<-13.7$ & -13.7 to 15.0 & $>15.0$ \\
\hline
\end{tabular}

The azimuthal (Yaw) value is used as the angle-separating value. After storing the initial Yaw data, the result is classified into toe-in and toe-out walking by 0 degree (stable walking). The change in angle of the sensor is shown in Figure 1, Yaw value is determined by the rotation of $\mathrm{z}$-axis. Top view of the foot is shown in right side of Figure 1.

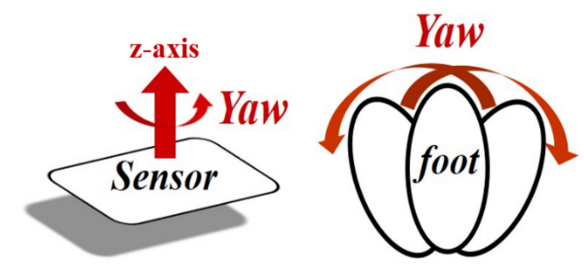

Figure 1. Yaw data of sensor for gait classification.

\section{Algorithm for Path Shift in Real Condition}

\subsection{Recognition of Switching Direction}

The user wears the device and moves on the various tracks. If the device is not reset after his turning, it will be confirmed as an abnormal gait. Therefore, after turning, the device should be reset. The flow chart for the algorithm used for switching direction is shown in Figure 2. It is assumed that 3 seconds is average for person to complete the turn around. After every 3 seconds, the algorithm was configured to check if a person was turning or not. In addition, since the reference of the corner is not fixed, the reference angle for judging the turning is assumed as 60 degrees. Where ' $\mathrm{t}$ ' is the time between 0 second and 5 seconds, $Y_{D}(t)$ is Yaw data (degree). $Y_{D 0}$ is standard Yaw data.

$$
Y_{D 0}= \begin{cases}Y_{D}(2), & t=2, \\ Y_{D}(5), & t=5 \text { and }\left|Y_{D}(5)-Y_{D 0}\right|>60, \\ Y_{D 0}, & \text { otherwise. }\end{cases}
$$

The algorithm of Figure 2 repeats Eq. (1).

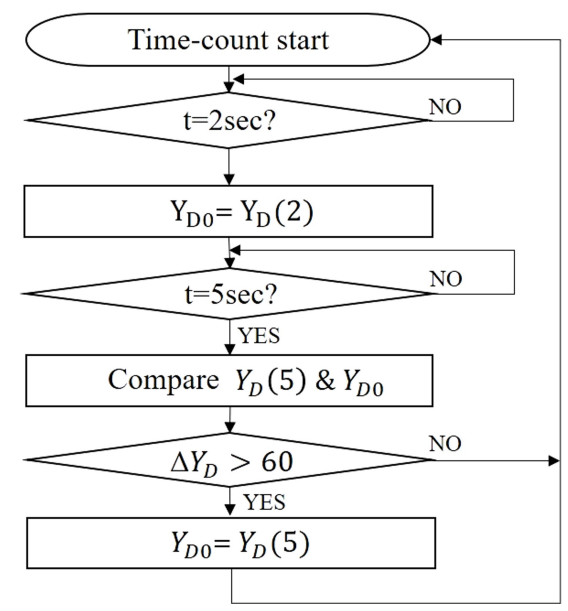

Figure 2. Algorithm for switching direction.

\subsection{Measuring the Number of Steps}

When the user is walking, movement of the ankle joint axis (transverse axis) occurs. This causes a characteristic change of Roll value from the sensor. This method helps to determine the number of walking steps. The Roll direction caused by the up-and-down movement of the ankle is shown in Figure 3. Roll value is determined by the rotation of $\mathrm{x}$-axis. Side view of the foot is shown in right side of Figure 2.

There is a difference between the foot height and the foot 


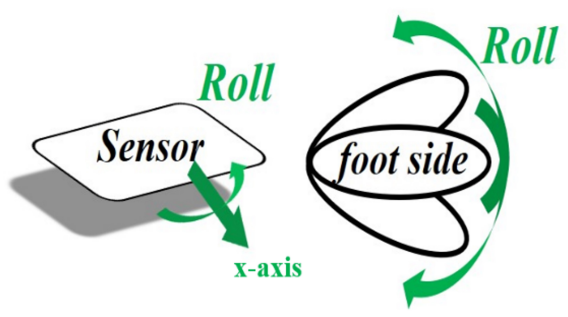

Figure 3. Roll data of sensor for measuring step number.

flexion for each user. If we take the initial Roll value as a reference, this will not be a problem. Additionally, Roll value while walking 20 steps with sneakers is demonstrated in Figure 4. There are three horizontal lines. The line from the top represents the $R_{M A X}, R_{W 0}, R_{M I N}$. These lines correspond to mid-stance state's: start term, initial Roll references and mid-stance state's: final term respectively. An idea has been derived from previous studies, estimates the stride length of a gait using an acceleration sensor [9]. The change in Roll value while walking 20 steps with slippers is presented in Figure 5. Even if watching ankles and shin movements, the results are similar [10 12].

$$
\begin{aligned}
& \text { Roll data }>R_{M A X}, \\
& \text { Roll data }<R_{M I N} .
\end{aligned}
$$

During a walk, if Eqs. (2) and (3) are satisfied, the number of steps will be accepted.

$$
\begin{aligned}
A_{R W} & =\frac{\frac{\left|R_{M A X}-R_{W 0}\right|}{2}+\frac{\left|R_{M I N}-R_{W 0}\right|}{2}}{2} ; \\
A_{R W} & \in \text { Sneakers; } \\
A_{R W} & \in \text { Slippers. }
\end{aligned}
$$

From Eq. (4), Eqs. (5) and (6) are obtained. $A_{M I N}$ is defined as 5.0 from Eq. (6), which is the minimum value from Eqs. (5) and (6). This is a value, to consider, a user having a small angle difference between the horizontal plane and the foot plane during his walking. It is recognized as a step, when the difference is more than the angle corresponding to $A_{M I N}$ on the basis of $R_{W 0}$ Eq. (7). $S_{N}$ is the step number to be measured, and $R_{\text {cur }}$ is the current Roll data. $f$ is the flag variable used to measure the maximum number of steps per time spent walking one step. Within the section that differs from the initial reference value of $R_{W 0}$ by as much as $A_{M I N}$, it was set to $f=0$ so that the walking distance would not be checked until it left the section again. The initial value is
$S_{N}=0$.

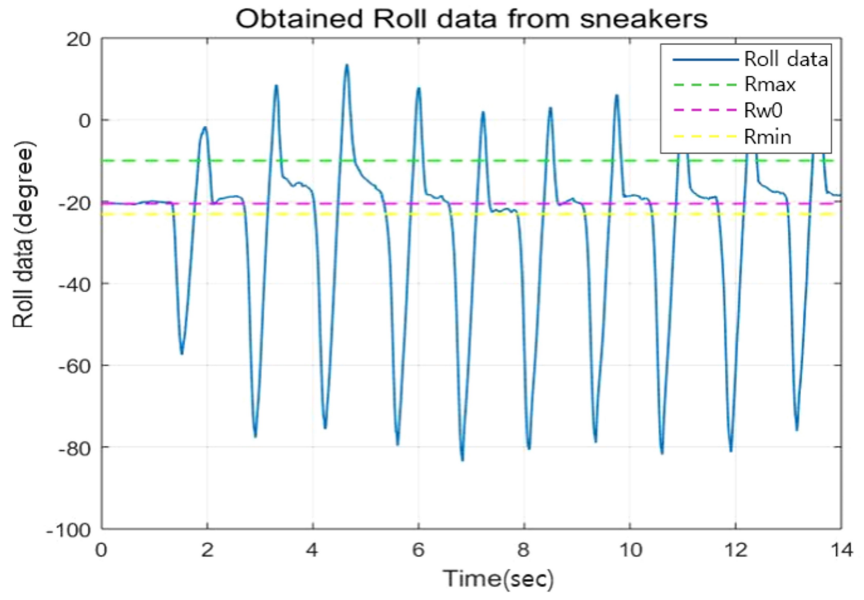

Figure 4. A variation of roll data graph about time (Sneakers).

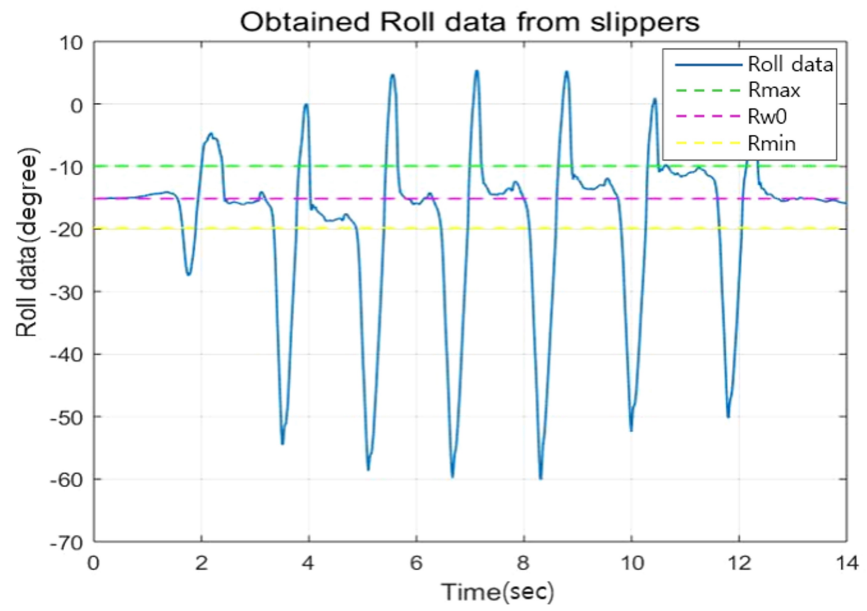

Figure 5. A variation of roll data graph about time (Slippers).

$$
S_{N}= \begin{cases}S_{N}+1, f=0, & \left(R_{W 0}-A_{M I N}<R_{c u r}\right. \\ & \left.<R_{W 0}+A_{M I N}\right) \text { and } f=1, \\ S_{N}, f=1, & \text { otherwise and } f=0 .\end{cases}
$$

Finally, it is possible to measure the number of individual steps for each foot. Figure 6 demonstrates a flow chart for measuring the number of steps.

\subsection{Walk Stop Recognition}

Walk stop is based on, when the user is stopped to walk motion pauses. This is detected at fixed time intervals, so that the LED 


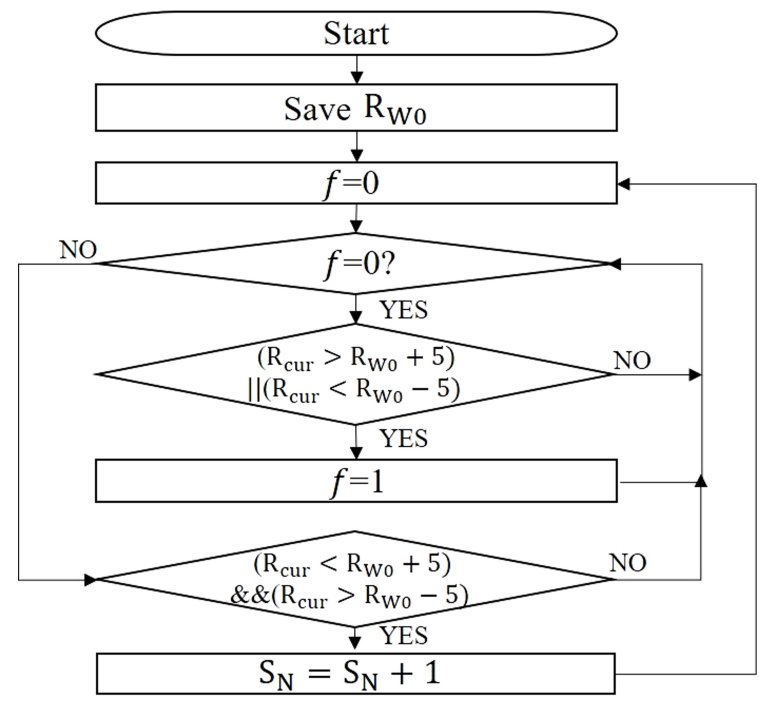

Figure 6. Algorithm for measuring step number.

feedback does not exist. The flow chart of the walking mode reset after a certain time when there is no change in the number of steps is presented in Figure 7. $S_{N p r e}$ is the stored number of steps when $\mathrm{t}$ is $5(\mathrm{sec})$ and the other variables are defined in Figure 7.

$S_{N \text { pre }}= \begin{cases}S_{N}, & t=5, \\ S_{N}, \quad Y_{D 0}=Y_{D}(2), & t=10 \text { and } S_{N p r e}-S_{N}=0, \\ S_{N \text { pre }}, & \text { otherwise. }\end{cases}$

The algorithm of Figure 7 repeats Eq. 87.

The value is stored at every 5 seconds is compared to the value every 10 seconds. If there is no difference in value, the device turned to reset mode.

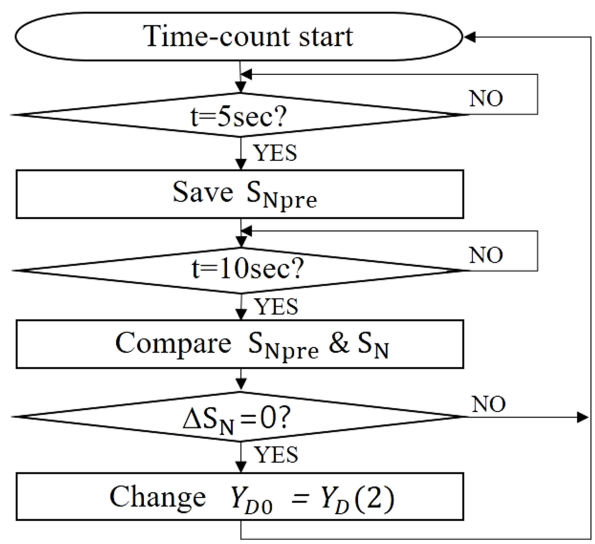

Figure 7. Reset algorithm after 10 seconds for pulling up.

\section{Experiment}

\subsection{Experimental Environment}

Based on the algorithm designed in the previous section, a wearable identification device (hereinafter referred to as device) is designed for checking the gait. The EBIMU-9DOFV3 AHRS sensor was used for measuring the Yaw and Roll data of the algorithm. Additionally, Neo Pixel LED was used to get visual feedback from the sensor. LED displays different 3 color according to the gait. Device's system demonstrated in Figure 8 . Figure 9 displays an example of the device, that can be attached to a variety of shoes. The color classification according to each gait is shown in Figure 10.

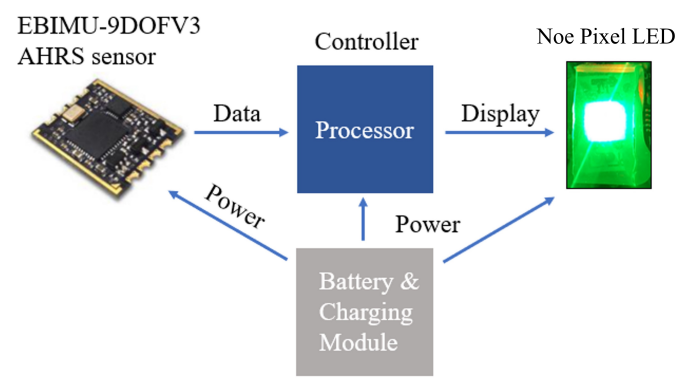

Figure 8. System illustration.

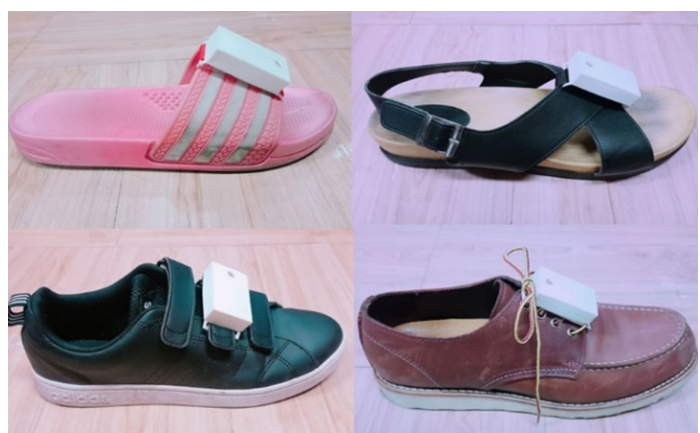

Figure 9. Detachable device on various shoe type.

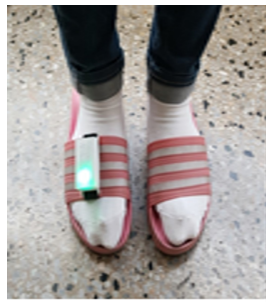

(a)

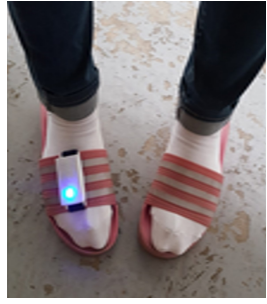

(b)

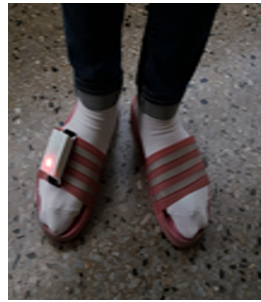

(c)
Figure 10. Color classification of a device according to gait: (a) green light for a steady walk, (b) blue light for toe-in gait, and (c) red light for toe-out gait. 


\subsection{Measuring Accuracy of Gait Classification}

For the gait classification of the male and female participants, three types of shoes were used to measure accuracy. The size of the shoes was chosen according to the size of the participants. The speed of the step was $4 \mathrm{~km} / \mathrm{hr}$. Table 2 provides information and results for experiments. For taking average, Eq. (9) is used. An explanation for the variables used in Eq. 99 is shown in Eq. (10). The average value of 35 measurements in 40 steps according to the shoe. For accurate results, each movement of the left foot and right foot was calculated as an independent step count. The measured value is the frequency at which the light of the sensor is changed during 40 steps.

$$
\begin{aligned}
& \text { Average }=\left(\frac{\sum^{35} L_{40}}{35 \times 40}\right) \times 100, \\
& L_{40}=\text { frequency of user's gait for } 40 \text { steps. }
\end{aligned}
$$

Table 2. Accuracy of device according to wearing various shoe type

\begin{tabular}{cccc}
\hline \multirow{2}{*}{$\begin{array}{c}\text { Walk } \\
\text { as usual }\end{array}$} & \multicolumn{3}{c}{$\begin{array}{c}\text { Average(\%) of the usual gait } \\
\text { when walking 40 steps }\end{array}$} \\
\cline { 2 - 4 } & Sneakers & Dress shoes & Slippers \\
\hline $\begin{array}{c}\text { Toe-out } \\
\text { walking } \\
\text { (male) }\end{array}$ & 87.29 & 80.76 & 88.29 \\
$\begin{array}{c}\text { Toe-out } \\
\text { walking } \\
\text { (female) }\end{array}$ & 97.00 & 94.29 & 96.00 \\
\hline
\end{tabular}

The two users show toe-out walking only in the swing phase. The frequency of specific gait between the shoes was similar. Therefore, this study demonstrates that it is not a gait classification system that can be applied to only running shoes, but a system that can be applied even when people wear various kinds of shoes.

\subsection{Step Count Measurement Accuracy}

Experiments on step count measurement accuracy were carried out by comparing, the actual number of walking, mobile application, and device. A woman with a $250 \mathrm{~mm}$ foot size experimented with a $250 \mathrm{~mm}$ size slippers. Tables 3 and 4 present the experimental results. Since the measurement error of the device depends on the speed of the stepping, so the measurement is based on the standard step and the slow step. For accurate results, each movement of the left foot and right foot was calculated as an independent step count. The average value of 30 measurements was applied. For accuracy, Eq.
(11) was applied. Test number of accuracy means the device measurements average where Tables 3 and 4 .

$$
\text { Accuracy }=100-\left(\frac{\mid \text { Step number-test number } \mid}{\text { Step number }} \times 100\right) \text {. }
$$

Table 3. Step count measurement accuracy when walking slowly (1 $\mathrm{km} / \mathrm{hr}$ )

\begin{tabular}{cccc}
\hline $\begin{array}{c}\text { Number of } \\
\text { steps }\end{array}$ & $\begin{array}{c}\text { Device } \\
\text { measurements } \\
\text { average }\end{array}$ & $\begin{array}{c}\text { Accuracy } \\
(\boldsymbol{\%})\end{array}$ & $\begin{array}{c}\text { Average } \\
(\boldsymbol{\%})\end{array}$ \\
\hline 10 & 10.1 & 99.0 & \\
20 & 19.97 & 99.85 & 99.17 \\
30 & 29.87 & 99.57 & \\
50 & 50.87 & 98.26 & \\
\hline
\end{tabular}

Table 4. Step count measurement accuracy when walking as usual (4 $\mathrm{km} / \mathrm{hr}$ )

\begin{tabular}{cccc}
\hline $\begin{array}{c}\text { Number of } \\
\text { steps }\end{array}$ & $\begin{array}{c}\text { Device } \\
\text { measurements } \\
\text { average }\end{array}$ & $\begin{array}{c}\text { Accuracy } \\
(\boldsymbol{\%})\end{array}$ & $\begin{array}{c}\text { Average } \\
(\boldsymbol{\%})\end{array}$ \\
\hline 10 & 10.17 & 98.3 & \\
20 & 20.07 & 99.65 & 99.17 \\
30 & 29.9 & 99.67 & \\
50 & 50.47 & 99.06 & \\
\hline
\end{tabular}

Compare with, to the health mobile application, Samsung Health, using the same conditions. The experiment was conducted with the mobile phone, Galaxy Note 5, which was in a pocket. Table 5 presents the number of steps measured by the health application. Table 5 shows the average value of 30 measurements. For accuracy, Eq. (11) was applied. Average value of accuracy is described in Table 5 .

Table 5. Step number accuracy of health application of phone when walking as usual $(4 \mathrm{~km} / \mathrm{h})$

\begin{tabular}{cccc}
\hline $\begin{array}{c}\text { Number of } \\
\text { steps }\end{array}$ & $\begin{array}{c}\text { Application } \\
\text { measurements } \\
\text { average }\end{array}$ & $\begin{array}{c}\text { Accuracy } \\
(\mathbf{\%})\end{array}$ & $\begin{array}{c}\text { Average } \\
(\mathbf{\%})\end{array}$ \\
\hline 10 & 9.16 & 91.61 & \\
20 & 18.97 & 94.84 & 95.36 \\
30 & 28.94 & 96.45 & \\
50 & 49.16 & 98.32 & \\
\hline
\end{tabular}

At a normal pace $(4 \mathrm{~km} / \mathrm{hr})$, the accuracy of the device was an average of $3.81 \%$ higher than the other health applications. At a 
slow pace $(1 \mathrm{~km} / \mathrm{hr})$, the health applications are not measurable.

\section{Conclusion}

Due to the lack and limitations of current gait correcting devices, this study proposes another solution. That offers, more accessibility as well portability. Furthermore, by developing a device based on these criteria, it is possible to obtain accurate results.

In this study, this algorithm was proposed to classify gait by compensating the defect of gait analysis using the sensors located in the insole. By developing the device based on it, it is always possible to check the gait of a person. By attaching the device to various shoes, it has become possible to measure gait without being limited to certain shoes.

The angle of toe-out walking is specified by foot angle. The angle of feet will change the style of walking. In the previous experiments, gait classification was carried out including swing phase and stance phase. Instead of this case, if the angle is checked only by the stance phase, it is expected to satisfy the gait classification criteria and show higher accuracy. In our following study, we will continue to modify the algorithm based on these results, which will provide more accurate data on the angle of foot and gait [13].

Currently, the device is still in its primitive stages and further development will be need to provide a smaller and more accessible, and aesthetically, pleasing design. This will allow users to become more inclined to use this device in their everyday life.

The device will be developed and improved as a device, considering the user's view through the miniaturization and advanced design. This will reduce the resistance of users to wear on the surface of the shoe.

Additional applications can also be developed to allow doctors and rehabilitation therapists to provide statistical data and give feedback on the patient's gait. Since the gait is digitized, the patient will be able to take care of the gait in everyday life and will proceed in a direction to help the quick correction.

The second thing is interest. For example, if the proposed device is developed for children, the LEDs of the device will be interesting to children's gait. So that it can be worn constantly, a gait corrective treatment can be performed in the form of a game.

If the development continues to make the device accessible and interesting, the research will continue with algorithm for a detachable and wearable walking care. These studies will have a significant impact on our lives.

\section{Conflict of Interest}

No potential conflict of interest relevant to this article was reported.

\section{Acknowledgments}

This research was supported by Basic Science Research Program through the National Research Foundation of Korea (NRF) funded by the Ministry of Science (2017R1D1A3B04031864).

\section{References}

[1] J. Shin, H. Lee, S. Kim, B. Bae, and J. Cho, "Visual feedback system design for gait management," in Proceedings of HCI Korea, Seoul, Korea, 2014, pp. 286-291.

[2] G. Lee, G. Yeom, J. Rhee, D. Jeong, and J. Cho, "Auditory feedback interface design for real-time gait management," in Proceeding of HCI Korea, Pyeongchang, Korea, 2017, pp. $458-461$.

[3] S. D. Min and C. K. Kwon, "Step counts and posture monitoring system using insole type textile capacitive pressure sensor for smart gait analysis," Journal of The Korea Society of Computer and Information, vol. 17, no. 8, pp. 107-114, 2012. https://doi.org/10.9708/jksci.2012. 17.8.107

[4] S. J. Yoon, S. H. Yoon, Y. G. Choi, and K. H. Kim, "A study on gait pattern using a smart insole," in Proceedings of 48th Summer Conference on the Korean Institute of Electrical Engineers, Busan, Korea, 2017, pp. 1402-1403.

[5] H. Kim, W. Park, N. Seo, and J. Kim, "Development of smart insole and mobile application for healthcare," in Proceedings of 2014 Symposium of the Korean Institute of Communications and Information Sciences, Seoul, Korea, 2014, pp. 321-322.

[6] S. M. Kim, D. H. Kim, B. H. Lee, D. H. Koo, and D. H. Lee, "mSiS: mobile smart insole system using pressure, gyroscope sensor," in Proceedings of Winter Conference on Korean Institute of Information Sciences and Engineers, Pyeongchang, Korea, 2015, pp.1651-1652. 
[7] D. Rosenbaum, "Foot loading patterns can be changed by deliberately walking with in-toeing or out-toeing gait modifications," Gait \& Posture, vol. 38, no. 4, pp. 10671069, 2013. https://doi.org/10.1016/j.gaitpost.2013.04.001

[8] O. B. Kwon, "Kinematic and kinetic analysis of the outtoeing gait pattern," M. S. thesis, Department of Physical Education, University of Hallym, Chuncheon, Korea, 2001.

[9] H. K. Kwak, S. H. Park, and C. W. Lee, "Design and implementation of pedestrian position information system in GPS-disabled area," Journal of the Korea AcademiaIndustrial Cooperation Society, vol. 13, no. 9, pp. 41314138, 2012. https://doi.org/10.5762/KAIS.2012.13.9.4131

[10] B. H. Kim, "Contact repulsion of robotic foot and its influence on knee and hip joints," Journal of Korean Institute of Intelligent Systems, vol. 23, no. 1, pp. 12-17, 2013. https://doi.org/10.5391/JKIIS.2013.23.1.12

[11] E. P. Doheny, T. G. Foran, and B. R. Greene, "A single gyroscope method for spatial gait analysis," in Proceedings of 2010 Annual International Conference of the IEEE Engineering in Medicine and Biology, Buenos Aires, Argentina, 2010, pp. 1300-1303. https://doi.org/10.1109/ IEMBS.2010.5626397

[12] J. S. Cho, S. I. Kang, K. H. Lee, S. H. Jang, I. Y. Kim, and J. S. Lee, "Implementation of gait analysis system based on inertial sensors," Journal of Rehabilitation Welfare Engineering \& Assistive Technology, vol. 9, no. 2, pp. 137-144, 2015.

[13] J. M. Kim, "The analysis of walking variables in normal and out-toed pedestrian according to height of shoe insole," M. S. thesis, Department of Physical Education, Kongju National University, Gongju, Korea, 2014.

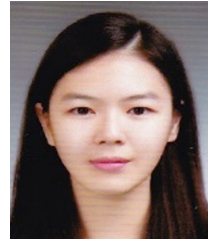

Nan-Hee Kim is undergraduate courses in Electronic Engineering from Yeungnam University in Korea. Her research interests include deep learning and control theory.

E-mail: kimnanhee97@naver.com

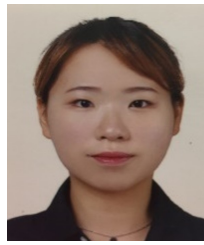

Ju-Hyung Lee is undergraduate courses in Electronic Engineering from Yeungnam University in Korea. Her research interests include optimization, image processing, and computer vision.

E-mail: nana970@naver.com

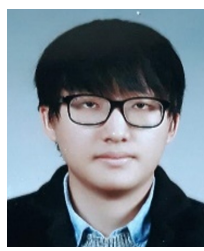

Seo-Jin Woo is undergraduate courses in Electronic Engineering from Yeungnam University in Korea. His research interest includes robotics.

E-mail: tiwls419@naver.com

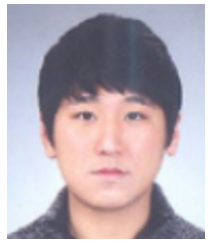

Dong-Lin Kwon received his B.S. and M.S. degrees in Electrical Engineering from Yeungnam University in 2015 and 2017, respectively. From 2017 until now, he has been worked in Kiturami Boiler Company. His research interests include robotics and designing controller.

E-mail: yjs2998@naver.com

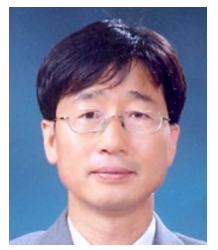

Suk-Gyu Lee received B.S. and M.S. degrees in Electrical Engineering from Seoul University, in 1979 and 1981. He completed his Ph.D. in Electrical Engineering from UCLA in 1990. From 1982 until now he is a Electrical Engineering professor in Yeungnam University in Korea. His research interests are control theory and robotics.

E-mail: sglee@ynu.ac.kr 